\section{Stress and the genesis of diabetes mellitus}

\section{in schizophrenia}

TIMOTHY G. DINAN

\section{METHOD}

A selective review was made of papers on Medline. Secondary sources such as book chapters were also searched.

\section{RESULTS}

\section{Stress endocrinology}

The endocrine stress system has two broad components with considerable central anatomic interconnection. The acute response to stress, which usually lasts a few minutes, consists of sympathetic adrenal-medullary (SAM) activation. The chronic stress response is mediated by the hypothalamic-pituitary-adrenal (HPA) axis. Cannon (1932) proposed the concept of homoeostasis, whereby bodily systems are regulated to maintain a steady state. Such a view has significantly influenced the development of modern integrative physiology. The SAM axis has a fundamental role in homoeostasis and is regulated at a pontine level by the locus coeruleus, a noradrenergic nucleus which provides a highly arborised neuronal network influencing levels of arousal and vigilance. The SAM system controls acute reaction to stress in what Cannon described as the 'fight or flight' response. He demonstrated that without a sympathetic nervous system an animal could survive in an unstressed environment; however, when subjected to stress, the animal could not mount basic physiological responses such as mobilisation of glucose. Activation of the SAM axis results in secretion of the catecholamines adrenaline and noradrenaline from the adrenal medulla, which is essentially an enlarged and highly specialised sympathetic ganglion. Because these catecholamines regulate acute responses they have short half-lives (1-3 min), with a high metabolic clearance rate and rapid degradation by catechol $\mathrm{O}$-methyltransferase, monoamine oxidase and aldehyde oxidase (Esler et al, 1990).

Adrenaline and noradrenaline exert their impact through $\alpha$-and $\beta$-adrenoceptors (Ahlquist, 1974). Adrenaline is most potent at $\beta_{1^{-}}$and $\beta_{2}$-receptors, with far less effect on $\alpha$-receptors, whereas noradrenaline is more potent at $\alpha$-receptors. The hyperglycaemic impact of the adrenomedullary hormone is mediated by adrenaline, which is a profoundly diabetogenic hormone. Adrenaline produces a hyperglycaemic effect in that it both stimulates hepatic glucose 
production and also limits glucose utilisation. The hepatic effect is mediated largely through $\beta$-adrenergic stimulation, although $\alpha$-adrenergic stimulation may have a part to play (Macdonald, 1999). The impact of adrenaline on glucose production is transient and takes place within minutes. The ability to limit glucose utilisation occurs predominantly through the $\beta$-receptor. As a result of this impact on glucose utilisation, sustained hyperadrenalism produces sustained hyperglycaemia.

Noradrenaline exerts hyperglycaemic actions when released from axon terminals of sympathetic post-ganglionic neurons. The liver has an important sympathetic innervation, and in animals when these sympathetic nerves are electrically stimulated a decrease in glycogen content is reported, together with increased hepatic glucose release, resulting in hyperglycaemia (Lautt, 1980). There is no evidence that this system is involved in the regulation of carbohydrate metabolism under normal circumstances, but it comes into play in situations of significant stress. Interestingly, Kjaer et al (1995) reported that the liver denervation that occurs with transplantation does not cause a gross alteration in carbohydrate metabolism. The primary metabolic impact of the SAM axis is control of fat metabolism (Niijima, 1989). Prolonged starvation and other significant stressors increase lipolysis through the SAM response mediated by $\beta$-adrenoceptors. In marked contrast, stimulation of $\alpha$-adrenoceptors inhibits lipolysis in adipose tissue. Although the sympathetic innervation of white adipose tissue mainly supplies the vasculature, in some areas there is direct innervation of the adipose cells. Overall, brown adipose tissue has a greater vascular supply and innervation than white tissue and a greater percentage of these cells are sympathetically innervated, with the metabolic effects again mediated through $\beta$-adrenoceptors. Stimulation of the sympathetic innervation of the pancreatic $\beta$-cells produces an inhibition of insulin release mediated by $\alpha$-adrenoceptors, probably of the $\alpha_{2}$ subtype. When the SAM system remains activated there is a reduction in the effectiveness of insulin to stimulate glucose uptake and utilisation. Such an impact is produced through the $\beta_{2}$-adrenoceptors and is mimicked by drugs such as salbutamol and terbutaline. High doses of $\beta_{2}$ agonists stimulate adipose tissue lipolysis and induce pancreatic glucagon secretion, which can lead to increased ketone production.

\section{Function of the SAM axis in schizophrenia}

No comprehensive study has used contemporary assays to examine SAM function in schizophrenia. Kemali et al (1985) examined catecholamine release over $24 \mathrm{~h}$ in patients with schizophrenia and a matched comparison group; the patients had been drug-free for a minimum of 2 weeks. Plasma noradrenaline levels were consistently elevated in the patients during the waking period but not during sleep. In a similar study, Barbeito et al (1984) found an elevation in catecholamine levels in both plasma and urine, and their data support the view that urinary catecholamine measurement could be used as a reliable assessment of SAM activity in schizophrenia. The finding is consistent with a report that unmedicated patients with schizophrenia have elevated levels of noradrenaline as well as heightened responsivity on measures of electromyographic activity, skin conductance and heart rate (Albus et al, 1982); the patients showed an attenuated response to the cold pressor test, noise and mental arithmetic stressors. A more recent study by Fleischhaker et al (1998) reported that response to clozapine in patients with treatment-resistant schizophrenia was associated with increases in adrenaline levels, a finding that might help to explain the pro-diabetic effect of this drug.

\section{Hypothalamic-pituitary-adrenal axis}

The ability to sustain a stress response is dependent on chronic activation of the HPA axis. Its major hormones are well defined and are easily assayed. The prohormone pro-opiomelanocortin is produced in the corticotrophs of the anterior pituitary and has a pivotal role in the axis. Its cleavage results in the production of adrenocorticotrophic hormone (ACTH), $\beta$-endorphin and several other biologically active peptides. The release from the adrenal cortex of cortisol, dehydroepiandrosterone (DHEA) and other steroids is stimulated by ACTH. Corticotrophinreleasing hormone $(\mathrm{CRH})$ and arginine vasopressin are the major secretagogues of the HPA stress system. Corticotrophinreleasing hormone, a 41 amino acid peptide originally discovered and sequenced by Vale et al (1981), is produced in the medial parvicellular neurons of the paraventricular nucleus of the hypothalamus. These neurons project to the external zone of the median eminence, where CRH is released into the portal vasculature to act on $\mathrm{CRH}$ type 1 receptors of the anterior pituitary. The hormone acts synergistically with arginine vasopressin in bringing about ACTH release from the corticotrophs. Following its identification in 1954, vasopressin, a nonapeptide, was considered to be the principal factor in the regulation of ACTH release, but with the subsequent elucidation of the structure of $\mathrm{CRH}$ and the domination of the 'one neuron - one transmitter' principle, the role of $\mathrm{CRH}$ came to supersede that of arginine vasopressin. It is now apparent that in stressfree situations $\mathrm{CRH}$ is the dominant regulator of the HPA axis, but with chronic stress many paraventricular neurons that normally produce $\mathrm{CRH}$ begin to co-express arginine vasopressin (Scott \& Dinan, 2002). In these circumstances arginine vasopressin plays an important part in sustaining HPA activation. The $\mathrm{CRH}_{1}$ receptor downregulates with increased production of $\mathrm{CRH}$, while increased production of arginine vasopressin upregulates the $V_{1 b}$ receptor. This latter effect is important in maintaining high cortisol output in the presence of a chronic stressor. In the absence of such a mechanism, adrenocortical activity will decrease over time and prevent an adequate biological response to the chronic stress.

The HPA axis is well characterised and has been investigated in a variety of central and peripheral disorders. In depression, HPA dysregulation has been extensively investigated, but in schizophrenia HPA function has received far less attention.

\section{Function of the HPA axis in schizophrenia}

Given that the onset of schizophrenia is frequently precipitated by stress and that relapse often takes place in a similar setting, it is surprising that the investigation of stress at a biological level has received so little attention in schizophrenia research. Walder et al (2000) assayed cortisol levels in multiple salivary samples from people with schizophrenia. This technique has the advantage of being stress-free, and as the cortisol in saliva is unbound to protein, the assay provides a measure of biological activity. They found that cortisol levels were significantly elevated, and that the greater the severity of symptoms the greater the elevation. The results support the view 
that the psychotic features of schizophrenia generate considerable biological stress. In a study of 53 patients taking medication, Kaneda et al (2002) found elevated levels of ACTH but failed to find abnormalities in plasma cortisol. Demonstrating differences in the latter usually requires multiple sampling, even in patients with overt Cushing's disease. The dexamethasone suppression test is a test of delayed feedback mechanisms in the HPA axis. In patients with predominantly positive symptoms a non-suppression rate of $56 \%$ was reported; patients with negative symptoms had a non-suppression rate of $53 \%$ (Pivac et al, 1997). These results are similar to those observed in major depression and are clearly different from those observed in healthy individuals. In a similar study Muck-Seler et al (1999) reported high baseline cortisol levels in schizophrenia with a non-suppression rate of $50 \%$, and Plocka-Lewandowska et al (2001) reported data suggesting that patients with schizophrenia who are suicidal are most likely to be non-suppressors. These studies support the view that defective HPA feedback mechanisms, possibly due to insensitive glucocorticoid receptors, may have a causative role. Levels of $\mathrm{CRH}$ in the cerebrospinal fluid have been investigated by Banki et al (1987): in patients with schizophrenia they found levels that were above normal, but not as high as those observed in patients with melancholic depression. The dexamethasone-CRH test has been applied to patients with schizophrenia (Heuser et al, 1994). This test involves the administration of dexamethasone at $23.00 \mathrm{~h}$ and the administration of $\mathrm{CRH}$ on the following day at $15.00 \mathrm{~h}$. Paradoxically, the dexamethasone pretreatment enhances the response to $\mathrm{CRH}$ in healthy individuals, and this enhancement is augmented in patients with major depression. Heuser et al (1994) found that patients with schizophrenia release more cortisol and ACTH after the dexamethasone-CRH test than do agematched healthy controls.

Few investigators have employed nonpharmacological strategies to activate the HPA axis in schizophrenia. Jansen et al (2000) used public speaking as a psychological stressor in patients with schizophrenia, and measured their salivary cortisol response to this stress. The patients showed a blunted response, in contrast to their response to the physical stress of cycling.

\section{CLINICAL IMPLICATIONS}

- Psychotic stress can produce a transient suppression of pancreatic $\beta$-cell function and alter insulin sensitivity.

- Cortisol has a pronounced antagonistic impact on insulin-mediated inhibition of hepatic glucose release while simultaneously decreasing glucose utilisation in muscle and reducing the binding affinity of insulin receptors.

Increase in the production of the diabetogenic hormones cortisol and adrenaline may help explain the increased tendency of patients with schizophrenia to develop diabetes.

\section{LIMITATIONS}

Many of the studies reported are characterised by small sample size.

- Studies of adrenaline production in schizophrenia are dated and made use of assays with low sensitivity and specificity by present standards.

- No large-scale prospective study examining stress hormones and glucose in schizophrenia has been published.

TIMOTHY G. DINAN, MD, PhD, DSc, FRCPsych, Department of Psychiatry, University College Cork; correspondence: GF Unit, Cork University Hospital,Wilton, Cork, Ireland. E-mail: t.dinan@ucc.ie

\section{Stress and glucose regulation}

There has been no systematic research to date exploring the endocrine response in people with schizophrenia exposed to a high-EE environment. This is surprising given the large number of studies published, especially in the UK, on high expressed emotion and schizophrenia. However, Shiloah et al (2003) examined $\beta$-cell function and insulin sensitivity using the homoeostasis model in non-diabetic patients with schizophrenia who had acute psychosis. A total of 39 individuals were assessed and the homoeostasis model assessment was based on two samples of glucose and insulin. Stress was assessed using the Clinical Global Impression (CGI) scale, which rates psychological stress as a score of 0-7 (Guy, 1976). On admission the mean CGI score of the patients was 5.3 (s.d. $=0.8$ ) and on discharge it was 1.6 (s.d.=0.7). Patients with the highest CGI scores on admission had the highest glucose and insulin levels. Mean $\beta$-cell function was lowest on admission $(96.8 \%$, s.d. $=33.2 \%)$ and highest at discharge $(134.4 \%$, s.d. $=60.0 \%)$. In marked contrast, mean insulin sensitivity was highest on admission $(101.7 \%$, s.d. $=36 \%)$ and had decreased significantly by discharge $(77.1 \%$, s.d. $=34.8 \%)$. However, insulin sensitivity inversely correlated with CGI score: a high CGI score was associated with low insulin sensitivity, and a low score was associated with high insulin sensitivity. The data support the view that psychotic stress produces a transient suppression of $\beta$-cell function and alters insulin sensitivity.

The impact of psychosis on glucose tolerance in drug-naïve patients with firstepisode schizophrenia has been reported by Ryan et al (2003), who compared 26 patients with schizophrenia and a similar number of control participants matched for age and gender. Four of the patients and none of the comparison group had an impaired fasting blood glucose concentration as defined by the American Diabetes Association (1997): $>6.1 \mathrm{mmol} / \mathrm{l}$ $(>110 \mathrm{mg} / \mathrm{dl})$ and $<7.0 \mathrm{mmol} / \mathrm{l}(<126 \mathrm{mg} / \mathrm{dl})$. Patients had higher mean fasting levels of glucose and insulin. Furthermore, they had elevated cortisol levels, raising the possibility that the dysregulation in glucose homoeostasis is secondary to overactivation of the HPA. 


\section{Effect of glucocorticoids}

Glucocorticoids inhibit insulin function in a variety of ways. Cortisol has a pronounced antagonistic impact on insulin-mediated inhibition of hepatic glucose release while simultaneously decreasing glucose utilisation in muscle and reducing the binding affinity of insulin receptors (Meyer \& Badenhoop, 2003). When patients are treated with glucocorticoids over long periods almost half of them develop deranged glucose metabolism, and in half of these patients this dysregulation persists even after reduction or withdrawal of the glucocorticoid. This situation is analogous to that occurring in schizophrenia, where glucose homoeostasis may be altered during acute episodes of illness and where the overall emergence of type 2 diabetes mellitus is higher than that seen in the general population.

\section{DISCUSSION}

A stress model of schizophrenia can help explain the tendency of people with this disorder to develop disturbance in glucose homoeostasis. Numerous studies report overactivation of the HPA axis with attenuated feedback inhibition and increased cortisol output. Although the SAM axis has been less extensively studied, the available evidence suggests that people with schizophrenia have heightened SAM responses with increased adrenaline output. Intermittent or sustained increase in the activity of these core endocrine stress systems with increase in the production of the diabetogenic hormones cortisol and adrenaline may help to explain the increased tendency of such patients to develop diabetes. Such a biological vulnerability would render individuals even more susceptible to the negative effects of poor diet and lack of exercise.

\section{REFERENCES}

Ahlquist, R. P. (1974) Adrenergic receptors: a persona and practical view. Perspectives in Biology \& Medicine, 16 119-122.

Albus, M., Ackenheil, M., Engel, R. R., et al (1982) Situational reactivity of autonomic functions in schizophrenic patients. Psychiatry Research, 6, 361-370.

American Diabetes Association (1997) Report of the Expert Committee on Diagnosis and Classification of Diabetes Mellitus. Diabetes Care, 20, II83-1197.

Banki, C. M., Bissette, G., Arato, M., et al (1987) CSF corticotrophin-releasing factor-like immunoreactivity in depression and schizophrenia. American journal of Psychiatry, 144, 873-877.

Barbeito, L., Lisa, A., Siveira, R., et al (1984) Resting urinary catecholamine exertion in schizophrenics: methodology and interpretation of results. Biological Psychiatry, 19, 1419-1425.

Brown, G. W., Birley, J. L. \& Wing, J. K. (1972) Influence of family life on the course of schizophrenic disorders: a replication. British Journal of Psychiatry, $\mathbf{1 2 1}$, $241-258$

Cannon, W. B. (1932) The Wisdom of the Body. New York: Norton.

Esler, M., Jennings, G., Meredith, I., et al (1990) Overflow of catecholamine neurotransmitter to the circulation: source, fate and function. Physiology Reviews, 70, 963-985.

Fleischhaker, C., Schultz, E. \& Remschmidt, H. (1998) Biogenic amines as predictors of response to clozapine treatment in early-onset schizophrenia. Journal of Psychiatric Research, 32, 325-333.

Guy, W. (1976) ECDEU Assessment Manual for Psychopharmacology. Revised DHEW Pub. (ADM). Rockville, MD: National Institute for Mental Health.

Heuser, I., Yassouridis, A. \& Holsboer, F. (1994) The combined dexamethasone/CRH test: a refined laboratory test for psychiatric disorders. Journal of Psychiatric Research, 28, 34I-256.

Jansen, L. M., Gispen-de Wied, C. C. \& Kahn, R. S. (2000) Selective impairment in the stress response in schizophrenic patients. Psychopharmacology, 149 319-325

Kaneda, Y., Fujii, A. \& Ohmori, T. (2002) The hypothalamic-pituitary-adrenal axis in chronic schizophrenic patients long-term treated with neuroleptics. Progress in Neuropsychopharmacology and Biological Psychiatry, 26, 935-938.

Kemali, D., Maj, M., Ariano, M. G., et al (1985) 24-hour plasma levels of prolactin, cortisol, growth hormone and catecholamines in schizophrenic patients. Neuropsychobiology, 14, 109-114.
Kjaer, M., Keiding, S., Engfred, K., et al (1995) Glucose homeostasis during exercise in humans with a liver or kidney transplant. American Journal of Physiology, 268, E636-E644.

Lautt, W. W. (1980) Hepatic nerves. A review of their function and effects. Canadian Journal of Physiology and Pharmacology, 58, II05-1123.

Macdonald, I. A. (1999) The influence of the autonomic nervous system on metabolic function. In Autonomic Failure: A Textbook of Clinical Disorders of the Autonomic Nervous System (4th edn) (eds C. J. Mathias \& R. Bannister), pp. 136-142. Oxford: Oxford University Press.

Meyer, G. \& Badenhoop, K. (2003) Glucocorticoidinduced insulin resistance and diabetes mellitus. Receptor-, postreceptor mechanisms, local cortisol action and new aspects of antidiabetic therapy Medizinische Klinik, 98, 266-270.

Muck-Seler, D., Pivac, N., Jakovljevic, M., et al (1999) Platelet serotonin, plasma cortisol and dexamethasone suppression test in schizophrenic patients. Biological Psychiatry, 45, 1433-1439.

Niijima, A. (1989) Nervous regulation of metabolism. Progress in Neurobiology, 33, 135-247.

Pivac, N., Muck-Seler, D. \& Jakovljevic, M. (1997) Platelet $5 \mathrm{HT}$ levels and hypothalamic-pituitaryadrenal axis activity in schizophrenic patients with positive and negative symptoms. Neuropsychobiology, 36 |9-2|.

Plocka-Lewandowska, M., Araszkiewicz, A. \& Rybakowski, J. K. (200I) Dexamethasone suppression test and suicide attempts in schizophrenic patients. European Psychiatry, 16, 428-431.

Ryan, M. C., Collins, P. \& Thakore, J. H. (2003) Impaired fasting glucose tolerance in first-episode, drugnaïve patients with schizophrenia. American Journal of Psychiatry, 160, 284-289.

Scott, L.V. \& Dinan, T. G. (2002) Vasopressin as target for antidepressant development: an analysis of the available evidence. Journal of Affective Disorders, 72 $|13-12|$

Shiloah, E., Witz, S., Abramovitch, Y., et al (2003) Effect of acute psychotic stress in nondiabetic subjects on $\boldsymbol{\beta}$-cell function and insulin sensitivity. Diabetes Care, 26, 1462-1467.

Vale, W., Spiess, J., Rivier, C., et al (198I)

Characterisation of a $4 \mathrm{I}$ residue ovine hypothalamic peptide that stimulates secretion of the corticotropin and beta-endorphin. Science, 213, 1394-1399.

Walder, D. J., Walker, E. F. \& Lewine, R. J. (2000) Cognitive functioning, cortisol release and symptom severity in patients with schizophrenia. Biological Psychiatry, 48, ||2|-|132. 